\title{
Multilayer metal/metal-oxide diffractive structure for photonic temperature sensing
}

\author{
Loukas Athanasekos, ${ }^{1,2, *}$ Miltiadis Vasileiadis,,${ }^{1,2}$ Anna Tsigara, ${ }^{3}$ Eliana Kaminska, ${ }^{4}$ Anna Piotrowska, ${ }^{4}$ \\ Dimitris Alexandropoulos, ${ }^{1}$ Michail M. Sigalas, ${ }^{1}$ and Nikos A. Vainos ${ }^{1,2}$ \\ ${ }^{1}$ Department of Materials Science, University of Patras, Rio, Patras 26500, Greece \\ ${ }^{2}$ National Hellenic Research Foundation, Theoretical and Physical Chemistry Institute, \\ 48 Vassileos Constantinou Avenue, Athens 116 35, Greece \\ ${ }^{3}$ Microsensors for Clinical Research and Analysis, Centre for Research in Electroanalytical Technology, \\ Department of Science, Institute of Technology Tallaght Dublin, Dublin 24 Ireland \\ ${ }^{4}$ Institute of Electron Technology, Aleja Lotnikow 32/46, 02-668, Warsaw, Poland \\ *Corresponding author: athanasekos@eie.gr
}

Received July 30, 2010; accepted October 4, 2010;

posted November 8, 2010 (Doc. ID 132532); published November 23, 2010

\begin{abstract}
We designed and fabricated multilayer metal/metal-oxide surface relief diffractive grating structures by growing alternating Pt and $\mathrm{SnO}_{\mathrm{x}}$ layers. Optical interrogation at $633 \mathrm{~nm}$ reveals the temperature dependence of their reflection and transmission diffractive effects. This function is explored here in the context of a remote, spatially localized, photonic temperature sensing operation, achieving sensitivity of $10 \%$ per ${ }^{\circ} \mathrm{C}$ for the zeroth-order in the transmission mode. The experimental demonstration is found to be in good agreement with the results of rigorous coupled wave analysis of the composite metal/metal-oxide element. @ 2010 Optical Society of America

OCIS codes: $\quad 050.1950,120.0280,120.6780,230.1950,280.4788,280.6780$.
\end{abstract}

Nanostructured photonic media offer a new potential in information and environmental technologies. Reversible refractive effects are, for example, observed upon environmental exposure and lead to physical and chemical sensing operations [1-3]. The latter are probed via interferometric and diffractive effects and used for the implementation of practical devices. In this context, although metals introduce loss, mixed metal/dielectric layered systems exhibit significant advantages in tuning the composition, spatially controlling the clustering, and, eventually, engineering the dielectric contrast, which enhances linear, nonlinear, or plasmonic responses [ $\underline{4}-\underline{6}]$. In a relevant effort, the interferometric behavior of a pulsed laser-deposited $\mathrm{Zn}-\mathrm{ZnO}-\mathrm{Zn}$ trilayer etalon structure was studied [7] with a $\sim 0.57 \%$ per ${ }^{\circ} \mathrm{C}$ response caused by the temperature dependence of the refractive index in the nonstoichiometric mesoscopic oxide.

In this Letter, we design, fabricate, and study, for the first time to our knowledge, a new type of surface relief metal/metal-oxide multilayer diffractive element. Its temperature sensitive diffraction is explored here in the context of spatially localized, "point," temperature sensing. In such operations, contact probe methods [8] technically fail due to thermal conduction, while spectral radiometry [9] has proved quite complex and expensive. Alternative optical methods using fiber Bragg gratings are intrusive, and temperature variations affect optical resonances, an effect enhanced by metal claddings $[10,11]$ and doping [12]. The proposed remote optical interrogation of a passive diffractive element provides an innovative, viable, low-cost sensing operation applicable in adverse and limited access environments.

Multilayered $\mathrm{Pt} / \mathrm{SnO}_{\mathrm{x}}$ diffractive structures have been designed by applying matrix methods and rigorous coupled wave analysis (RCWA) for optimum performance at $\lambda=633 \mathrm{~nm}$ assuming nominal refractive index values. The tin oxide refractive index value was set as $n_{\left(\mathrm{SnO}_{\mathrm{x}}\right)} \sim 2$, and zero extinction was considered, while the complex refractive index values of Pt for wavelengths around $633 \mathrm{~nm}$ were taken from [13]. The primary assumptions ignore possible material modification by nonstoichiometric growth or metal in-diffusion at the metal-oxide interfaces that would lead to variable structural properties.

Our design considers only variations in the real part of the refractive index, $n_{\left(\mathrm{SnO}_{\mathrm{x}}\right)}$, those being expected to be the most susceptible to temperature or other environmental changes. The multilayer structure design of Fig. 1 comprises a series of alternating metal and oxide layers grown on silica, denoted by $\mathrm{SiO}_{2} / \mathrm{Pt} / \mathrm{SnO}_{\mathrm{x}} / \mathrm{Pt} / \mathrm{SnO}_{\mathrm{x}} / \mathrm{Pt}$, corresponding to semi-infinite silica $/ 100 \mathrm{~nm} / 170 \mathrm{~nm} / 5 \mathrm{~nm} /$ $170 \mathrm{~nm} / 5 \mathrm{~nm}$. The fabricated diffractive elements have a period of $4 \mu \mathrm{m}$, a value limited by fabrication capability. The aim is, however, toward a small grating period for obtaining an appreciable separation of diffraction orders, with a consequent enhancement of diffraction efficiency and sensor sensitivity. The $4 \mu \mathrm{m}$ grating period chosen is thus a trade-off between optimized performance and fabrication complexity. An interrogating laser beam is

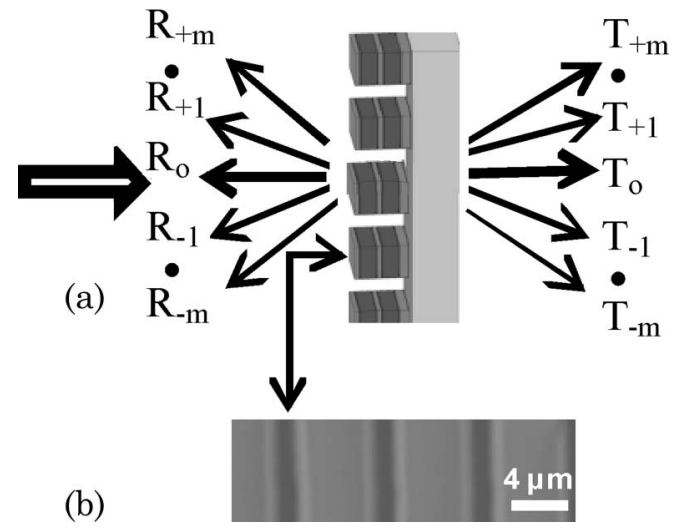

Fig. 1. (a) Schematic outline of structure design and the interrogation concept. (b) Scanning electron micrograph of a segment of the multilayer diffractive element. 
incident on the structure from the left, and the relevant diffracted light beams are indicated by referring to $R_{m}$ and $T_{m}(m=0, \pm 1, \pm 2, \pm 3 \ldots)$, parameters denoting the $m$ th-diffraction-order intensity reflection and transmission coefficients, respectively.

Figure 2 depicts the calculated zero-order transmission $\left(T_{0}\right)$ together with the zero- $\left(R_{0}\right)$ and first-order $\left(R_{+1}\right)$ reflection at $633 \mathrm{~nm}$ as a function of the typically anticipated $\mathrm{SnO}_{\mathrm{x}}$ refractive index. The RCWA is applied for the actual experimental conditions. A nearly zero $R_{0}$ value is reached at $n_{\left(\mathrm{SnO}_{\mathrm{x}}\right)}=2.2$. Similarly, the first-order reflection, $R_{+1}$, attains also a minimum of $\sim 0.3 \%$ at $n_{\left(\mathrm{SnO}_{\mathrm{x}}\right)}=2.1$. On the other hand, $T_{0}$ increases with $n_{\left(\mathrm{SnO}_{\mathrm{x}}\right)}$ from $12 \%$ to $20 \%$. The present analysis shows that the nominal $n_{\left(\mathrm{SnO}_{\mathrm{x}}\right)}=2.0$ offers a maximum $R$ and $T$ sensitivity for refractive index variations, and it fully corroborates the original choice of design parameters.

The representative structure has been fabricated by growing the structure $\mathrm{SiO}_{2} / \mathrm{Pt} / \mathrm{SnO}_{\mathrm{x}} / \mathrm{Pt} / \mathrm{SnO}_{\mathrm{x}} / \mathrm{Pt}: \mathrm{SiO}_{2} /$ $100 / 170 / 5 / 170 / 5$ (all values in $\mathrm{nm}$ ). Pt layers were deposited by DC sputtering using a metallic target. Tin oxide $\left(\mathrm{SnO}_{\mathrm{x}}\right)$ layers were also grown by reactive $\mathrm{DC}$ sputtering from a solid $\mathrm{Sn}$ metal target in $\mathrm{O}_{2} / \mathrm{Ar}$ plasma. The deposition conditions have been tuned to achieve nonstoichiometric growth of the tin oxide in order to take advantage of the refractive index variability produced by structural imperfections. A series of previous experiments analyzed the conditions for nonstoichiometric mixed polycrystalline/amorphous growth. Oxygen partial pressure was kept at $\sim 2 \times 10^{-3}$ mbar, while sputtering power was $75 \mathrm{~W}$. The oxide layer thickness and refractive index have been monitored on line. Off-line ellipsometric measurements have been performed and used for process calibration. Following multilayer deposition, the structures were patterned by photolithography and etched by inert $\mathrm{Ar}^{+}$ion etching to form the relief pattern and avoid reactive contamination, a process imposing fabrication limitations. A scanning electron micrograph of the final device is shown in Fig. 1 . The originally designed $50 \%$ pattern duty cycle is affected by the sputteretching process.

The effects on the refractive index of the oxide structure have been explored here by varying the temperature $\left(\Theta{ }^{\circ} \mathrm{C}\right)$ to alter the diffraction conditions. Similar nonstoichiometric materials have been explored in previous

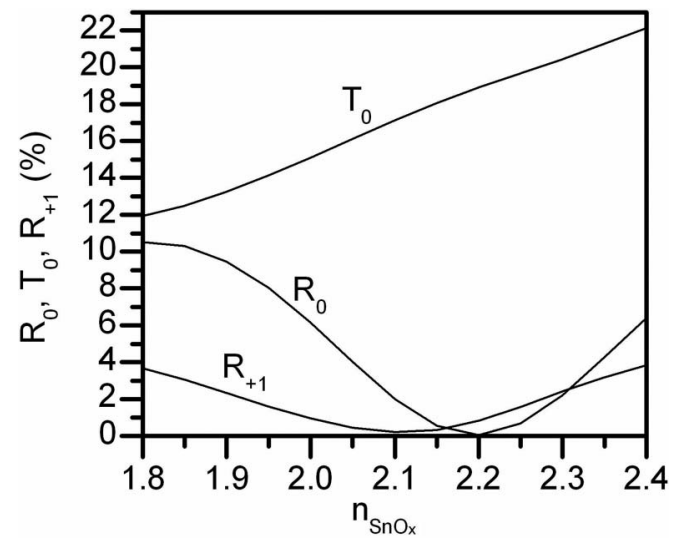

Fig. 2. Theoretical behavior of the $T_{0}, R_{0}$, and $R_{+1}$ at the $633 \mathrm{~nm}$ wavelength as a function of the refractive index, $\mathrm{SnO}_{\mathrm{x}}$, of the nonstoichiometric tin oxide. works [14], and strong dependence of refractive index on temperature has been observed. We note here, however, that the present structure is a composite multilayer with anticipated diffusive interfaces. Gradient indices are produced upon growth, and, therefore, measurements on the specific oxide material cannot be performed. However, in the present structure, the refractive index variation is directly translated to the optical $R$ and $T$ parametric changes observed by implementing the interrogation concept of Fig. 1. To evaluate the temperature sensitivity experimentally, the structure was placed in a thermoelectrically controlled miniature open oven. A Type K thermocouple was thermally coupled close to the grating structure and is used to reference the temperature, though some error is expected. A He-Ne laser emitting $5 \mathrm{~mW}$ at $\lambda=633 \mathrm{~nm}$ is used, and various diffracted orders were monitored in parallel by using four power meter heads (Newport model 2832-C Dual Channel) connected to a LabView data acquisition system. The laser beam incidence angle was set at $19.4^{\circ}$ to normal in order to facilitate convenient positioning of the optics. A reference beam was always simultaneously recorded to compensate for laser power variations. A systematic deviation is arising, however, due to temporal lag of the thermocouple response in continuous recordings.

Simultaneous real-time monitoring was performed for $R_{0}, T_{0}$, and $R_{+1}$ for ascending and descending temperature cycles between $20^{\circ} \mathrm{C}$ and $60^{\circ} \mathrm{C}$, as depicted in Fig. 3. A considerable dependence of $R_{0}, T_{0}$, and $R_{+1}$ on temperature is recorded, while a similar behavior was also observed for the higher diffraction orders both in transmission and reflection. While the design refractive index of $n_{\mathrm{SnO}_{\mathrm{x}}}=2.0$ is used for maximum reflection sensitivity, temperature cycling provides a real estimate of $T_{0}, R_{0}$, and $R_{+1}$ by comparing theory (Fig. 2) and experiments (Fig. 3). A best fit is thus obtained for $n_{\left(\mathrm{SnO}_{\mathrm{x}}\right)}$ between 2.10 and 2.35, proved by the existence of reflectance minima in the temperature range of $40^{\circ} \mathrm{C}-50^{\circ} \mathrm{C}$ and the appearance of two nodes between the $R_{0}$ and $R_{+1}$, in full agreement with the theoretical prediction. By comparison with theory, these latter nodes correspond to

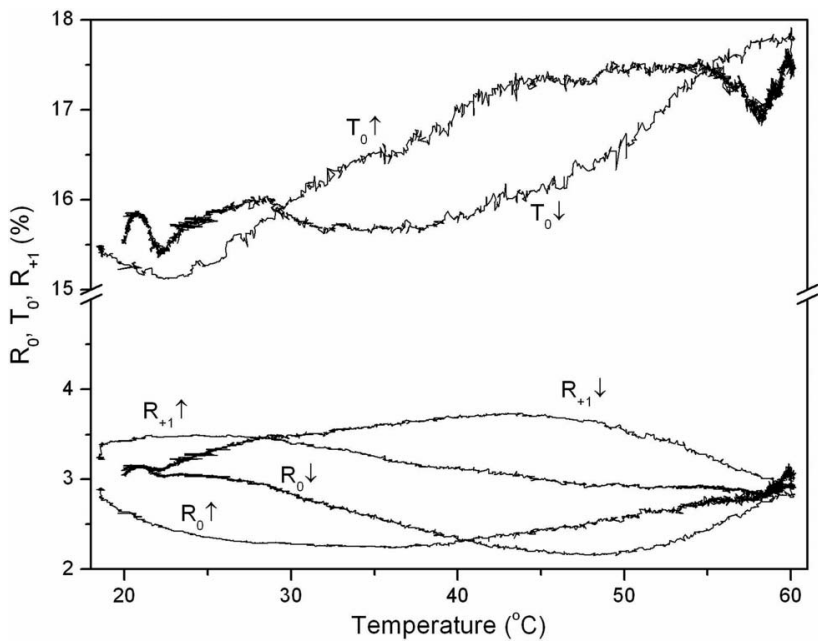

Fig. 3. Experimental behavior of three important parameters $R_{0}, T_{0}$, and $R_{+1}$ for ascending ( $\left.\uparrow\right)$ and descending $(\downarrow)$ temperatures $\Theta^{\circ} \mathrm{C}$. The observed crossing of $R_{0}$ and $R_{+1}$ is in full agreement with theory. 
the values $n_{\mathrm{SnO}_{\mathrm{x}}} \sim 2.17$ and $n_{\mathrm{SnO}_{\mathrm{x}}} \sim 2.32$ attained, respectively, at $\Theta \sim 23^{\circ} \mathrm{C}$ and $\Theta \sim 60^{\circ} \mathrm{C}$. A certain hysteresis in the response is observed leading to an open loop in the ascending trace, which may be justified by a certain lag of the reference thermocouple recording and heat diffusion through the metal cage. The transmission $T_{0}$ values and time recordings also agree very well with the theoretical prediction at a less than $10 \%$ difference. This optical method provides more confident results because the thermocouple probe physical contact usually affects the measurement. The refractive index $n_{\left(\mathrm{SnO}_{\mathrm{x}}\right)}$ of the grown oxide is found to be considerably higher than the nominal crystalline. This is justified by material imperfection and possible metal in-diffusion affecting nanocomposition, and, thus, some effects of absorption cannot be excluded. The structural imperfections result in a different refractive index from the nominal single crystal value of 2.0 and yield higher refractive index variations with temperature. An imperfect material is thus preferable in this application.

Considering the temperature variation, $\Delta \Theta^{\circ} \mathrm{C}$, the sensitivity, $S$, of the potential reflective sensor device may be defined as the variation of the diffraction efficiency per ${ }^{\circ} \mathrm{C}, S_{R+1}=\Delta R_{+1} /\left(\Delta \Theta \cdot R_{+1}\right)$. Fitting the linear sections of the recording curves (Fig. 3) provides an estimate of $S \sim\left(0.0262 \pm 2.96 \times 10^{-4}\right)^{\circ} \mathrm{C}^{-\mathrm{T}}$ or $\sim 2.62 \% /{ }^{\circ} \mathrm{C}$. A zeroorder reflected beam is found for $R_{0}: S_{R 0} \sim(0.02587 \pm$ $\left.3.81 \times 10^{-4}\right)^{\circ} \mathrm{C}^{-1}$. This reflectance sensitivity is improved $\times 4.5$ as compared to the zinc-based etalon scheme of [7]. Furthermore, the zero-order transmitted beam $T_{0}$ gives $S_{T 0} \sim\left(0.1 \pm 1.6 \times 10^{-3}\right)^{\circ} \mathrm{C}^{-1}$, thus indicating that the transmission mode may be preferable due to our original assumption. The estimated values of $n_{\left(\mathrm{SnO}_{\mathrm{x}}\right)}=2.10-2.32$ for the range $\Theta \sim 20^{\circ} \mathrm{C}-60^{\circ} \mathrm{C}$ would lead to an optimal device. It is emphasized here that the experimentally observed and theoretically analyzed behavior is solely attributed to the thermally induced refractive and possibly additional absorptive changes, rather than the thermal expansion effects, which have a negligible impact. In addition, it has to be noted that after testing a variety of samples, a slightly different performance with the same trends is observed, indicating the repeatability of results.

In conclusion, a novel layered nanocomposite diffractive device is designed, fabricated, and studied. Its tem- perature sensitive behavior is found to be in excellent agreement with its theoretical performance estimated by RCWA. The produced device functions with a high temperature sensitivity of $\sim 10 \%$ per ${ }^{\circ} \mathrm{C}$. This new approach establishes new concepts for photonic remote point sensing and would lead to further applications in information optics and photonics.

The authors would like to acknowledge the contribution and useful discussions of Alessio Perrone.

\section{References}

1. A. Meristoudi, L. Athanasekos, M. Vasileiadis, S. Pispas, G. Mousdis, E. Karoutsos, D. Alexandropoulos, H. Du, A. Tsigara, K. Kibasi, A. Perrone, and N. A. Vainos, J. Opt. A: Pure Appl. Opt. 11, 034005 (2009).

2. M. Vasileiadis, L. Athanasekos, A. Meristoudi, D. Alexandropoulos, G. Mousdis, E. Karoutsos, A. Botsialas, and N. A. Vainos, Opt. Lett. 35, 1476 (2010).

3. A. Tsigara, G. Mountrichas, K. Gatsouli, A. Nichelatti, S. Pispas, N. Madamopoulos, N. A. Vainos, H. L. Du, and F. Roubani-Kalantzopoulou, Sens. Actuators B Chem. 120, 481 (2007).

4. D. Owens, C. Fuentes-Hernandez, and B. Kippelen, Thin Solid Films 517, 2736 (2009).

5. C. Min, P. Wang, C. Chen, Y. Deng, Y. Lu, H. Ming, T. Ning, Y. Zhou, and G. Yang, Opt. Lett. 33, 869 (2008).

6. R. J. Blaikie, L. Lin, R. J. Reeves, and J. Roger, Int. J. Nanotechnol. 6, 222 (2009).

7. N. A. Vainos, A. Tsigara, J. Manasis, A. Giannoudakos, G. Mousdis, N. Vakakis, M. Kompitsas, A. Klini, and F. Roubani-Kalantzopoulou, Appl. Phys. A 79, 1395 (2004).

8. L. Michalski, K. Eckersdorf, J. Kucharski, and J. McGhee, Temperature Measurement (Wiley, 2001).

9. W. Ross McCluney, Introduction to Radiometry and Photometry (Artech, 1994).

10. L. Gang-Chih, L. Wang, C. C. Yang, M. C. Shih, and T. J. Chuang, IEEE Photon. Technol. Lett. 10, 406 (1998).

11. X. C. Li, F. Prinz, and J. Seim, Smart Mater. Struct. 10, 575 (2001).

12. G. Brambilla, "High-temperature fiber Bragg grating thermometer," Electron. Lett. 38, 954 (2002).

13. E. D. Palik, Handbook of Optical Constants of Solids (Academic, 1998).

14. G. Koundourakis, C. Rockstuhl, D. Papazoglou, A. Klini, I. Zergioti, N. A. Vainos, and C. Fotakis, Appl. Phys. Lett. 78, 868 (2001). 